\title{
A simple device for multiplex ELISA made from melt-extruded plastic microcapillary film
}

Article

Accepted Version

Manuscript plus supplementary data

Edwards, A. D., Reis, N. M., Slater, N. K. H. and Mackley, M. R. (2011) A simple device for multiplex ELISA made from meltextruded plastic microcapillary film. Lab on a Chip, 11 (24). pp. 4267-4273. ISSN 1473-0197 doi:

https://doi.org/10.1039/C0LC00357C Available at https://centaur.reading.ac.uk/24356/

It is advisable to refer to the publisher's version if you intend to cite from the work. See Guidance on citing.

Published version at: http://dx.doi.org/10.1039/C0LC00357C

To link to this article DOI: http://dx.doi.org/10.1039/C0LC00357C

Publisher: Royal Society of Chemistry

All outputs in CentAUR are protected by Intellectual Property Rights law, including copyright law. Copyright and IPR is retained by the creators or other copyright holders. Terms and conditions for use of this material are defined in the End User Agreement.

www.reading.ac.uk/centaur 
Central Archive at the University of Reading

Reading's research outputs online 


\title{
A simple device for multiplex ELISA made from melt-extruded plastic microcapillary film
}

Alexander D. Edwards ${ }^{¥ 1,2} *$, Nuno M. Reis ${ }^{¥ 1,3}$, Nigel K. H. Slater ${ }^{1}$, and Malcolm R. Mackley ${ }^{1}$

${ }^{1}$ Department of Chemical Engineering and Biotechnology, University of Cambridge, New Museums Site, Pembroke Street, Cambridge CB2 3RA, UK.

${ }^{2}$ Reading School of Pharmacy, Whiteknights, PO Box 224, Reading, RG6 6AD, UK.

${ }^{3}$ Current address: Department of Chemical Engineering, University of Loughborough, LE11 3TU, UK. Current email: n.m.reis@lboro.ac.uk

$¥$ These authors contributed equally to this work.

*CORRESPONDING AUTHOR:

Email: a.d.edwards@reading.ac.uk

Telephone: +44 (0) 1183784253

Fax +44 (0) 1183784703

\section{Technical Note}

\begin{abstract}
We present a simple device for multiplex quantitative enzyme-linked immunosorbant assays (ELISA) made from a novel melt-extruded microcapillary film (MCF) containing a parallel array of $200 \mu \mathrm{m}$ capillaries along its length. To make ELISA devices different protein antigens or antibodies were immobilised inside individual microcapillaries within long reels of MCF extruded from fluorinated ethylene propylene (FEP). Short pieces of coated film were cut and interfaced with a pipette, allowing sequential uptake of samples and detection solutions into all capillaries from a reagent well. As well as being simple to produce, these FEP MCF devices have excellent light transmittance allowing direct optical interrogation of the capillaries for simple signal quantification. Proof of concept experiments demonstrate both quantitative and multiplex assays in FEP MCF devices using a standard direct ELISA procedure and read using a flatbed scanner. This new multiplex immunoassay platform should find applications ranging from lab detection to point-of-care and field diagnostics.
\end{abstract}

\section{KEYWORDS}

Multiplexed, Immunoassay, micro-engineered, melt extrusion, fluoropolymer, Microcapillary film, ELISA

\section{Background}

Immunoassay is a core biochemical technique for the detection and quantification of molecules in biological samples. As the number of important analytes grows, and the number of measurements required expands, there is an increasing drive to simultaneously measure multiple analytes within a single sample, a method referred to as multiplex immunoassays. The measurement of a panel of biomarkers is increasingly required in diagnostics and there is a growing need to conduct multiplex immunoassays outside the laboratory either in the field or at the point of care ${ }^{1-3}$. Assay miniaturisation is therefore required to reduce volumes and achieve faster assay times, ease of use and portability. A plethora of microfluidic technologies have been explored ranging from the use of individual capillaries ${ }^{4-6}$ through to fully integrated systems containing complex microchannels ${ }^{1,7-10}$. In all cases, significant challenges include reducing device manufacturing costs, incorporating antibodies/antigens onto a detection surface, and signal detection. 
Immunoassays have been conducted in individual capillaries for several decades ${ }^{11}$, but recent developments have focussed on multiplexing, integrating fluidics, and improving signal detection within capillaries ${ }^{6}$. To conduct multiplex immunoassays, multiple individual capillaries coated with different antibodies or antigens can be connected to a single sample feed ${ }^{4,5}$ or multiple bands can be coated within a single capillary by photolithographic or micro-syringe methods ${ }^{12,13}$. Optical detection is particularly challenging because individual capillaries represent cylindrical lenses when viewed from the side. To overcome this, the capillary body can be used as an optical waveguide analogous to an optical fibre ${ }^{13}$, or converted substrate can be eluted into a flowthrough optical sensor ${ }^{4}$.

Many different microfluidic immunoassay devices have also been developed using microchannels formed in situ ranging from portable fully integrated microfluidic devices driven by syringes ${ }^{3}$ or capillary action ${ }^{2}$ to discs in which fluid movement is controlled by centrifugal force ${ }^{14-16}$. Although lab methods allow rapid prototyping of microfluidic devices, mass fabrication is more challenging leading to interest in simpler materials such as paper ${ }^{17}$ and development of advanced manufacturing methods ${ }^{3,10}$. Furthermore, even after fabrication, labile and costly antibodies or antigens must somehow be immobilised onto the detection surface within the device $^{7-9}$. Methods to achieve this include depositing antibodies onto microchannels prior to assembly ${ }^{2,7}$, introducing microparticles coated with detection reagents into the device ${ }^{14,18,19}$, and chemical methods such as DNA-directed assembly ${ }^{20}$. Finally, low-cost, sensitive and portable detection methods are also vital for signal measurement in microfluidic devices ${ }^{21}$; approaches include using solid state components (e.g. LEDs, CCD arrays) ${ }^{21}$, lasers $^{16}$, colour amplification for visual readout ${ }^{3}$ or non-optical methods such as electrochemical ${ }^{8}$ or magnetic ${ }^{22}$ detection.

In this paper we propose for the first time using plastic microcapillary film (MCF), a novel cheap microengineered material, for conducting multiplex immunoassays, as an alternative to individual capillaries or in situ formed microchannels. MCF are long melt extruded plastic films containing a parallel array of microcapillaries with controlled size and shape, introduced during extrusion by air injection ${ }^{23,24}$ (Fig. 1A). We present proof-of-concept data demonstrating quantitative and multiplex ELISA in MCF, and highlight possible advantages of this approach.

\section{Materials and Methods}

\subsection{Reagents and Materials}

The MCF studied here produced from fluorinated ethylene propylene (FEP MCF), and containing 10 capillaries with a mean diameter of $206 \pm 12.2 \mu \mathrm{m}$ and external dimensions of $4.5 \pm 0.1 \mathrm{~mm}$ wide by $0.6 \pm 0.05 \mathrm{~mm}$ thick (Fig. 1A), was produced by Laminar Dielectrics Ltd (Billingshurst, West Sussex, UK). Purified mouse IgG, mouse and goat serum, horseradish peroxidase conjugated anti-mouse IgG- (HRP-anti-mIgG), Alkaline Phosphatase conjugated anti-mouse IgG (AP-anti-mIgG), FLAG peptide, and mouse monoclonal anti-FLAG peptide (anti-FLAG), Tween 20, phosphate buffer saline pH7.4 (PBS), protease-free bovine serum albumin (BSA), SigmaFast OPD substrate tablets, and Nunc Maxisorp ELISA plates were from Sigma-Aldrich (Dorset, UK). Recombinant Hepatitis B Core Antigen (rHB-CAg), and mouse monoclonal anti-HB CAg (anti-HB CAg) were from Abcam (Cambridge, UK). HRP-conjugated anti-mouse IgG1 detection antibody was from Invitrogen (Paisley, UK). Fluorescein diphosphate (FDP) substrate (Cambridge Biosciences, Cambridge UK) was used at $20 \mu \mathrm{M}$ dissolved in $50 \mathrm{mM}$ Tris $\mathrm{pH} 9.0,10 \mathrm{mM}$ Glycine, $10 \mathrm{mM} \mathrm{MgCl}_{2}$. Washing solution was $0.05 \% \mathrm{v} / \mathrm{v}$ Tween 20 in PBS (PBS-T) and blocking solution was $1 \% \mathrm{w} / \mathrm{v}$ BSA in PBS or $1 \% \mathrm{w} / \mathrm{v}$ BSA plus $1 \% \mathrm{v} / \mathrm{v}$ goat serum in PBS.

\subsection{ELISA procedures}

To coat with antigen, $1 \mu \mathrm{g} / \mathrm{ml}$ of recombinant HB CAg in PBS was either filled into all capillaries within $1 \mathrm{~m}$ of FEP MCF, or 50ul/well was added to microtitre ELISA plates (MTP) and incubated overnight at $4^{\circ} \mathrm{C}$. MCF and MTP were then washed and blocked for at least $2 \mathrm{~h}$ at room temperature with $1 \%$ BSA plus $1 \%$ goat serum in PBS. To simulate seropositive sera with defined amounts of reactivity to HB CAg, normal mouse serum was spiked with known concentrations of a mouse IgG1 monoclonal antibody against HB CAg. A high-titre serum 
sample contained $50 \mu \mathrm{g} / \mathrm{ml}$ monoclonal anti-HB CAg antibody, and a low-titre sample contained $2 \mu \mathrm{g} / \mathrm{ml}$ monoclonal anti-HB CAg. Positive samples and a control sample were diluted 1:20 followed by 4-fold serial dilutions, and either aspirated into 30mm long MCF test strips or $80 \mu 1$ per well added to MTP in duplicate. After at least $1 \mathrm{~h}$ incubation at room temperature, MCF strips and MTP were washed 3 times and HRP anti-mouse IgG1 detection antibody at 1:3000 in PBS containing 1\% BSA + 1\% goat serum added. Between solutions the FEP MCF test strips were not emptied. After at least 45 minutes incubation with detection antibody at room temperature, MCF strips and MTP were washed extensively and OPD substrate added. Measurement of absolute anti-HB CAg concentration was compared in FEP MCF vs MTP using a similar procedure, except antigen was coated at $10 \mu \mathrm{g} / \mathrm{ml}$, block buffer was $1 \%$ BSA in PBS without goat serum, samples were diluted in $1 \%$ BSA in PBS without serum, and the detection antibody was HRP-anti-mIgG at 1:5000.

For signal detection, after 20-40 minutes of incubation with OPD, FEP MCF devices were scanned with a HP ScanJet 4050 Photo Scanner in transmittance mode (1,200-2,400 dpi resolution). Images were post-processed with Image J software ${ }^{25}$ to determine the absorbance in each capillary for the blue channel that gave strongest absorbance for the converted OPD substrate; image processing is described in supplementary methods. MTP were measured using a plate reader after $1 \mathrm{~h}$ incubation, either at $450 \mathrm{~nm}$ without stop solution or at $490 \mathrm{~nm}$ after adding $3 \mathrm{M} \mathrm{HCl}$. Absorbance values expressed as $\mathrm{cm}^{-1}$ were calculated relative to pathlengths of $0.02 \mathrm{~cm}$ for MCF and $0.3 \mathrm{~cm}$ for MTP to allow direct signal comparison.

To demonstrate multiplex immunoassays with fluorescent signal detection, the 10 capillaries within $5 \mathrm{~m}$ of FEP MCF were each individually coated by injection with PBS (negative control), 10 $\mu \mathrm{g} / \mathrm{ml}$ mouse-IgG (positive control), $10 \mu \mathrm{g} / \mathrm{ml}$ Hepatitis B core antigen protein or $10 \mu \mathrm{g} / \mathrm{ml}$ FLAG peptide in PBS in the indicated pattern followed by blocking of all capillaries with PBS/BSA. Individual 50-mm long pieces were cut and loaded with one of three test samples containing PBS alone, anti-HB CAg, or anti-FLAG peptide monoclonal antibodies and incubated for $1 \mathrm{~h}$. After washing, the pieces were incubated with AP-anti-mIgG followed by washing and addition of the fluorescent substrate FDP, and then scanned by confocal microscopy after 15 min incubation and scored as positive or negative; examples of images are shown in Fig. S3D.

\section{Results and Discussion}

\subsection{Producing Simple Microfluidic Devices from Melt-Extruded Microcapillary Film}

MCF was extruded from a highly transparent fluoropolymer, fluorinated ethylene propylene (FEP), with dimensions, surface characteristics, and optical properties suited to producing immunoassay devices (Fig. 1A). In order to adapt this material for conducting multiplex ELISA, whilst maintaining the benefits of the extruded manufacturing process and the simplicity of a plastic film, a two stage process was devised (Fig. 1). Firstly, antigens (for direct ELISA) or capture antibodies (for sandwich ELISA) were immobilised in bulk, with each capillary coated with different antigens/antibodies, within the capillaries of a long piece of MCF (e.g. 5 metres), conveniently wound on a reel (Fig. 1B). Blocking was also completed in bulk. Secondly individual assay pieces (e.g. 20-50mm in length) were cut from the reel of bulk coated MCF (Fig. 1C). In this way, hundreds of multiplex test strips can be produced in a single batch. Individual assay pieces were each fitted with a single feed for uptake of sample and detection solutions into all capillaries (Fig. 1D). A cross-sectional diagram of the resultant device is illustrated in Fig. 1F.

Given the hydrophobic surface properties of FEP, protein antigens and antibodies were simply immobilised by direct adsorption onto the inner surface of capillaries. Crucially, coating was found to be homogeneous along the length of a long MCF reel, demonstrated by direct detection of immobilised antibody in a test strip taken from the inlet (distance $0 \mathrm{~m}$ ) and outlet (distance $5 \mathrm{~m}$ ) of a $5 \mathrm{~m}$ long piece of FEP MCF (Fig. S1). Thus any convenient length of MCF can be coated in one batch, depending on the desired number of assay strips and the length of strip used. No difference was seen when coating lengths varying from $10 \mathrm{~cm}$ to $5 \mathrm{~m}$ (data not shown).

Two different simple fluid handling techniques were used to fill capillaries within MCF. To fill different solutions in different capillaries- for example to make multiplex assay strips (Fig. 1B) - individual capillaries were filled by simply injecting individual capillaries using a fine needle $(31 \mathrm{G})$ and syringe. Alternatively, all 
capillaries in a given piece of MCF were filled simultaneously with the same fluid by connecting one end of the test strip to a pipette or syringe using a $20-30 \mathrm{~mm}$ piece of $3 \mathrm{~mm}$ internal diameter silicone tubing pushed 5-10mm over the end of the MCF test strip and sealed by clamping with a tubing clamp or simply pinching between finger and thumb. The other end was immersed in the appropriate liquid in a reagent well (Fig. 1D, F). By aspirating measured volumes using a pipette or syringe, solutions were drawn up into all capillaries from the reagent well. A solution can be aspirated into a set of 8 FEP MCF assay strips manually using a single pipette within 2-3 minutes; more samples can be processed using multichannel pipettes (data not shown). Although silicone tubing is a simple and low-cost method for interfacing with pipettes, we also prototyped simple moulded push-fit seals customised to interface with other fluid handling devices such as syringes (Fig. 1E). Preliminary studies with dye solutions indicated a minimum volume of $30 \mu 1$ was required to equally fill the 10 capillaries in a $50 \mathrm{~mm}$ piece of MCF from a single microtitre plate well using silicone tubing and a pipette (data not shown); customised reagent wells and moulded push-fit seals would reduce volumes further.

Although cheap to produce (see supplementary results for cost estimates) and simpler to manufacture than in situ formed microchannels, using extruded material does not allow the integration of multiple steps possible with complex microfluidic devices ${ }^{1,2,7-9}$. However, this disadvantage has been overcome with individual capillary assays by integrating with other fluid handling components ${ }^{4,5}$. Similarly, microparticles are useful for immunoassays but difficult to process, a difficulty that has been overcome by incorporation into microfluidic devices $^{14,18,19}$. We propose that short MCF strips could be integrated into more complex fluid handling devices in a similar manner.

\subsection{Unique Optical Properties of FEP MCF}

A major barrier to the use of individual fused silica or plastic microcapillaries for immunoassays has been the difficulty of optically interrogating the contents due to the cylindrical capillary geometry and the refractive index of the capillary wall. The capillary represents two concentric cylindrical lenses with distortion caused by refraction at both the air to capillary surface interface, and also usually at the inner surface to water interface. In contrast, the FEP MCF used in the current study has two unique features that are ideal for optical signal detection by simple transmittance imaging (Fig. 1G). Firstly the flat parallel faces of the MCF provide a short path length through the film wall, with no curvature to refract the light path. Secondly, FEP has a refractive index of 1.338 which is very close to water (1.333), resulting in minimal optical refraction at the water:capillary wall interface and thus no distortion is caused by the capillaries when filled with aqueous solutions. Thus although capillaries are clearly visible when FEP MCF is air filled (Fig 1A), they completely disappear when filled with aqueous solutions (Fig. 1H). Refractive index matching using FEP for undistorted optical detection has been previously exploited to image microbial growth on packed beds ${ }^{26}$. To demonstrate the importance of its unique flat-sided geometry for simple optical detection of coloured substrate, FEP MCF was shown to have superior transparency to different sized individual, cylindrical FEP capillaries produced from the same fluoropolymer (Fig. S2A vs $\mathrm{B}, \mathrm{C})$. To demonstrate the importance of refractive index matching that of water, FEP MCF was shown to have superior transparency to other MCF with similar geometry but melt extruded from a range of different plastics with differing refractive indexes (Fig. S2A vs D-H).

In the present proof-of-principle study, two different optical detection methods were used. As an example of the simplest detection method needed, some experiments were conducted using a flatbed scanner in transmission mode to detect a colorimetric ELISA substrate (Fig. 1H, 2, S3B). The optical clarity could be exploited with other detection devices, for example CCD cameras, photodiode arrays, CMOS sensors, or even with suitable background illumination and holder - a smartphone camera. Other optical immunoassay signals could be measured such as chemiluminescence and fluorescence, and a fluorescent ELISA substrate was detected using confocal microscopy (Fig S1, S3D). The use of an off-the-shelf consumer flatbed scanner offers advantages over the measurement of converted substrate in a flow cell following elution from individual assay capillaries ${ }^{4}$, and over the customised optical detectors needed to measure light emission from capillary ends utilising waveguide effects ${ }^{13}$ or to analyse multi-capillary cassettes ${ }^{5}$. Furthermore, colour changes within MCF are clearly visible to the naked eye, allowing visual readout without the signal amplification described recently for field clinic microfluidic devices ${ }^{3}$. 


\subsection{Quantitative ELISA in FEP MCF Devices}

Having developed a method for making devices from FEP MCF and established its favourable optical properties, it was essential to demonstrate that quantitative, sensitive and multiplex immunoassays could be conducted in these devices. Three proof-of-principle direct ELISA assays were completed to demonstrate quantitative assays in real biological fluids (serum), to compare sensitivity with microtitre plates (MTP), and to illustrate multiplexed detection.

To test if relevant biological fluids could be quantitatively analysed using FEP MCF, a simple direct ELISA (Fig S3A) was conducted to detect mouse anti-HB CAg antibodies in parallel in both FEP MCF devices and MTPs. For simplicity and to determine inter-capillary variation, singleplex assay strips were made with the same antigen adsorbed in all capillaries. High or low amounts of monoclonal anti-HB CAg were spiked into serum samples, and compared with a control serum with no added anti-HB CAg. Seroreactivity against HB CAg was then measured using a conventional endpoint titre protocol, whereby these three serum samples were serially diluted and tested for reactivity to adsorbed antigen. An intense yellow colour indicating OPD substrate conversion was seen as increased amounts of anti-HB CAg were present in samples (Fig.S3B). The strongest absorbance by OPD was seen in the blue channel (Fig.S3B), and thus to quantify the signal the maximum blue light absorbance was determined for each capillary. As expected, absorbance increased when more anti-HB CAg was present and decreased with dilution confirming that quantitative direct ELISA is feasible for analysis of real biological fluids using FEP MCF, with conventional assay chemistry and read using a flatbed scanner (Fig. 2A, B). The pattern of absorbance seen with the three titrated mouse sera was broadly similar with FEP MCF and MTP, suggesting similar assay performance to conventional MTP ELISA (Fig. 2A, B). Due to the high concentrations of $\operatorname{IgG}$ in serum, at low dilutions control sera containing no specific antibodies gave a background signal; this was higher in microtitre plates than in FEP MCF devices (Fig. 2 A, B), demonstrating that FEP MCF gives no additional background compared to conventional MTP. However, when antibody against HB CAg was present in the serum samples, far higher signals were detected at higher dilutions. Furthermore the absolute absorbance values measured in FEP MCF were significantly higher than those observed in MTP (Fig. 2A, B), but in contrast the endpoint titre at which signal dropped below a threshold for the two positive sera was somewhat lower for FEP MCF compared to MTP, indicated some reduction in sensitivity in this assay. In this experiment, identical reagent conditions - optimised for MTP - were used in MCF; however, increased assay sensitivity should be possible in MCF by optimising reagent concentrations and improving scanner sensitivity.

To further explore the relative sensitivity of FEP MCF vs MTP assays without the complication of background signal caused by high levels of IgG present in whole serum, a direct ELISA was also conducted measuring fixed concentrations of monoclonal anti-HB CAg diluted without serum. Again, the intensity of yellow colour increased with incremental anti-HB CAg concentration (Fig. S3B). At concentrations over 100ng/ml antiHB CAg, the absorbance signal became saturated and non-linear, but below 40ng/ml a clear linear relationship between concentration and absorbance was seen (Fig. 2C, D, S3B). Again, a similar pattern of absorbance with varying concentration was seen with both FEP MCF and MTP, although as before higher absolute absorbance values were seen in FEP MCF than in the MTP assay. The limit of detection of anti-HB CAg with the FEP MCF was approximately $1 \mathrm{ng} / \mathrm{ml}$ which is identical to the sensitivity observed for MTP (Fig. 2C, D).

Note that in these experiments, the incubation times used for FEP MCF were kept identical to the standard MTP assay. However, in common with other microfluidic devices ${ }^{1,7-9,21}$, reduced diffusion distances should allow reduction in incubation times by 10-20-fold compared to MTP. For example, sandwich immunoassays were completed within 30 minutes in $320 \mu \mathrm{m}$ internal diameter individual capillaries ${ }^{4}$. Indeed, preliminary tests suggested that in FEP MCF, incubation times of 10 minutes give similar assay performance to $2 \mathrm{~h}$, in contrast to a significant reduction in signal strength observed with 10 minute incubations in MTP (data not shown). Similarly, sample and reagent volume requirement are reduced in MCF compared to MTP.

\subsection{Multiplex ELISA in FEP MCF Devices}

For proof-of-concept of multiplex IA, antibodies against two different antigens (HB CAg and the FLAG peptide) were measured using a simple direct ELISA process (Fig. S3C,D), each in triplicate within three 
different capillaries. Duplicate positive and negative control capillaries were also included resulting in assay strips that measure 4 'assay channels'. The antigens, plus positive (coating with mouse IgG) and negative (uncoated) controls, were filled into different capillaries of a $5 \mathrm{~m}$ reel of FEP MCF in the pattern illustrated in Table 1 and Fig. S3D. After coating and blocking, individual $50 \mathrm{~mm}$ test strips were then filled with three samples containing different antibodies (control, anti-HB CAg, anti-FLAG, Fig. S3D), followed by detection with AP-anti-mIgG and the fluorescent substrate FDP. As expected, low fluorescence levels were observed in the two negative control capillaries, and high fluorescence was seen in the positive controls, regardless of the sample used (Table 1 and Fig. S3D). In contrast, increased fluorescence was detected with the HB CAg coated capillaries only when the sample contained anti-HB CAg, and similarly the FLAG coated capillaries gave strong positive signal only with the sample containing anti-FLAG antibody (Table 1 and Fig. S3D). Some background signal was observed (especially with the detection of antigen (c) in sample (3) in one capillary); this was due to excess detection reagent concentrations and was eliminated by subsequent optimization (data not shown).

Although some technologies allow the detection of very large panels of analytes, for the majority of diagnostic assays between 2 and 10 analytes are typically measured ${ }^{3,5}$. The current FEP MCF extrusion can detect 8 different agents (disregarding the outer 2 capillaries; Fig. S2), which is ideally suited to these requirements; however MCF with 19 capillaries are routinely extruded ${ }^{24}$, giving scope for more analytes or multiple replicate measurements of the same analytes.

\section{Conclusions}

We present a new concept for a multiplex immunoassay platform based on the multi-channel, microengineered material FEP MCF. With further development and optimisation, this platform should offer the following features and benefits: 1) miniaturised for rapid and low volume assays; 2) simplicity of immobilising a panel of antibodies or antigens onto the detection surface; 3) simple, cheap and potentially portable optical signal detection; and 4) simple and cost-effective manufacturing process. The flat geometry of the plastic film combined with the optical clarity of FEP provides an opportunity for simple optical signal detection, for example using a flatbed scanner or smartphone camera, or using a simple customised detector made from low-cost LED and CCD or CMOS sensor components. Optical detection also allows use of conventional ELISA reagents and existing assay chemistry. We present proof-of-principle data demonstrating that quantitative, multiplex ELISA can be conducted using FEP MCF and quantified using a flatbed scanner, bringing for the first time the benefits of meltextrusion as a method for manufacturing microfluidic devices, combined with simple optical detection, to the field of immunoassays. Future studies will build on these observations by optimising conditions, determining assay speed and robustness, and developing more sophisticated FEP MCF devices combined with portable signal detection systems. These studies should lead to the development of a range of scalable, cost-effective multiplex immunoassay tools for applications from laboratory research to portable or point-of-care diagnostics.

\section{ACKNOWLEDGMENTS}

The authors are grateful to Patrick Hester from Lamina Dielectrics Ltd for providing the FEP MCF material. ADE and NMR are grateful to the Technology Strategy Board, the EPSRC and the European Commission (Marie Curie programme) for financial support. We are grateful to Ricardo Guraieb Chahín for helpful discussions and comments on the manuscript.

\section{REFERENCES}

1. P. Yager, T. Edwards, E. Fu, K. Helton, K. Nelson, M. R. Tam and B. H. Weigl, Nature, 2006, 442, 412418.

2. L. Gervais and E. Delamarche, Lab Chip, 2009, 9, 3330-3337.

3. C. D. Chin, T. Laksanasopin, Y. K. Cheung, D. Steinmiller, V. Linder, H. Parsa, J. Wang, H. Moore, R. Rouse, G. Umviligihozo, E. Karita, L. Mwambarangwe, S. L. Braunstein, J. van de Wijgert, R. Sahabo, J. E. Justman, W. El-Sadr and S. K. Sia, Nat Med, 2011, 17, 1015-1019.

4. S. Koch, H. Wolf, C. Danapel and K. A. Feller, Biosens Bioelectron, 2000, 14, 779-784.

5. E. Yacoub-George, W. Hell, L. Meixner, F. Wenninger, K. Bock, P. Lindner, H. Wolf, T. Kloth and K. A. Feller, Biosens Bioelectron, 2007, 22, 1368-1375. 
6. C. Mastichiadis, A. E. Niotis, P. S. Petrou, S. E. Kakabakos and K. Misiakos, Trac-Trends in Analytical Chemistry, 2008, 27, 771-784.

7. A. Bange, H. B. Halsall and W. R. Heineman, Biosensors \& Bioelectronics, 2005, 20, 2488-2503.

8. T. G. Henares, F. Mizutani and H. Hisamoto, Analytica Chimica Acta, 2008, 611, 17-30.

9. S. Choi, M. Goryll, L. Sin, P. Wong and J. Chae, Microfluidics and Nanofluidics, 2011, 10, 231-247.

10. C. Rivet, H. Lee, A. Hirsch, S. Hamilton and H. Lu, Chemical Engineering Science, 2011, 66, 1490-1507.

11. M. de Frutos, S. K. Paliwal and F. E. Regnier, Anal Chem, 1993, 65, 2159-2163.

12. C. Mastichiadis, S. E. Kakabakos, I. Christofidis, M. A. Koupparis, C. Willetts and K. Misiako, Anal Chem, 2002, 74, 6064-6072.

13. P. S. Petrou, S. E. Kakabakos, I. Christofidis, P. Argitis and K. Misiakos, Biosens Bioelectron, 2002, 17, 261-268.

14. B. S. Lee, J. N. Lee, J. M. Park, J. G. Lee, S. Kim, Y. K. Cho and C. Ko, Lab Chip, 2009, 9, 1548-1555.

15. R. Gorkin, J. Park, J. Siegrist, M. Amasia, B. S. Lee, J. M. Park, J. Kim, H. Kim, M. Madou and Y. K. Cho, Lab Chip, 2010, 10, 1758-1773.

16. N. Honda, U. Lindberg, P. Andersson, S. Hoffmann and H. Takei, Clin Chem, 2005, 51, 1955-1961.

17. A. W. Martinez, S. T. Phillips, Z. Nie, C.-M. Cheng, E. Carrilho, B. J. Wiley and G. M. Whitesides, Lab on a Chip, 2010.

18. C. T. Lim and Y. Zhang, Biosens Bioelectron, 2007, 22, 1197-1204.

19. M. Ikami, A. Kawakami, M. Kakuta, Y. Okamoto, N. Kaji, M. Tokeshi and Y. Baba, Lab Chip, 2010, 10, 3335-3340.

20. H. Schroeder, M. Adler, K. Gerigk, B. Muller-Chorus, F. Gotz and C. M. Niemeyer, Anal Chem, 2009, 81, 1275-1279.

21. F. B. Myers and L. P. Lee, Lab on a Chip, 2008, 8, 2015-2031.

22. J. Kim, E. C. Jensen, M. Megens, B. Boser and R. A. Mathies, Lab Chip, 2011.

23. B. Hallmark, M. R. Mackley and F. Gadala-Maria, Advanced Engineering Materials, 2005, 7, 545-547.

24. D. I. Medina, B. Hallmark, T. D. Lord and M. R. Mackley, Journal of Materials Science, 2008, 43, 52115221.

25. M. D. Abramoff, P. J. Magelhaes and S. J. Ram, Biophotonics International, 2004, 11, 36-42.

26. A. P. Leis, S. Schlicher, H. Franke and M. Strathmann, Applied and environmental microbiology, 2005, 71, 4801-4808.

TABLE 1

\begin{tabular}{llccc}
\hline \multirow{2}{*}{$\begin{array}{c}\text { Capillary } \\
\text { number }\end{array}$} & Coating antigen & $\begin{array}{c}\text { Sample 1 } \\
\text { buffer control }\end{array}$ & $\begin{array}{c}\text { Sample 2 } \\
\text { anti-HB CAg }\end{array}$ & $\begin{array}{c}\text { Sample 3 } \\
\text { anti-FLAG }\end{array}$ \\
\hline 1 & Negative control & - & - & - \\
2 & Positive control- mIgG & + & + & + \\
3 & Negative control & - & - & - \\
4 & Positive control- mIgG & + & + & + \\
5 & Hepatitis B Core Ag & - & + & - \\
6 & FLAG peptide & - & - & + \\
7 & Hepatitis B Core Ag & - & + & + \\
8 & FLAG peptide & - & - & $-/+$ \\
9 & Hepatitis B Core Ag & - & + & + \\
10 & FLAG peptide & - & - &
\end{tabular}

\section{FIGURES}




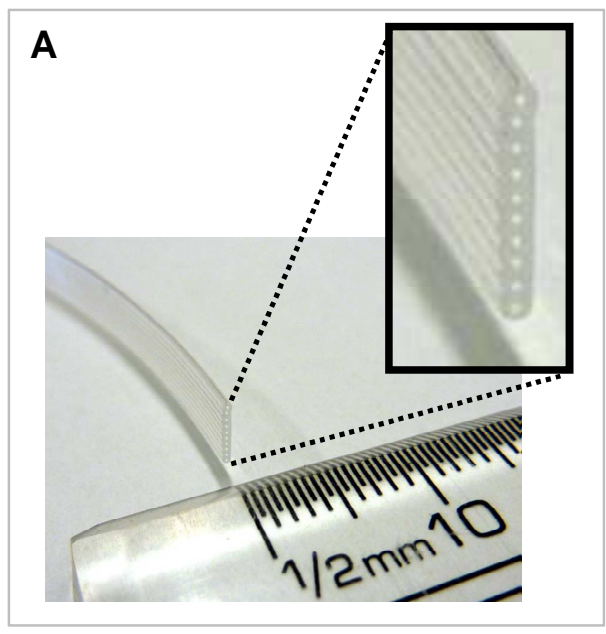

B Bulk coat

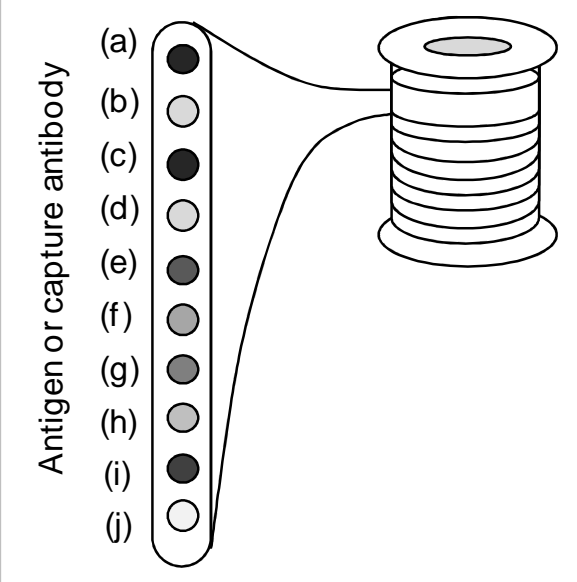

C Cut into assay strips

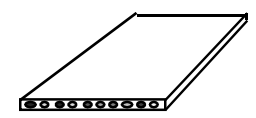

(a) - (j)
D
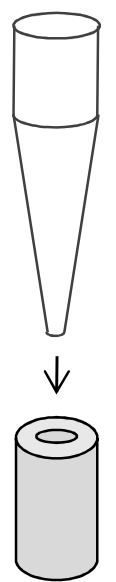

Silicone

tubing

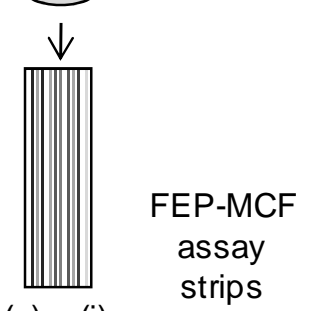

(a) - (j)

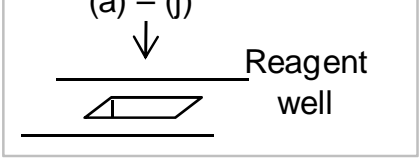

E

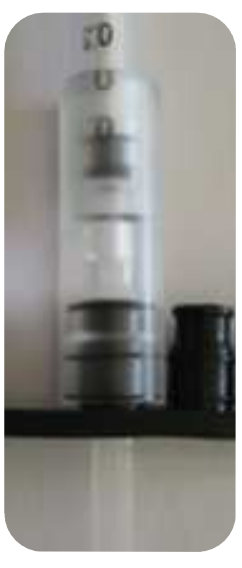

$\mathbf{F}$

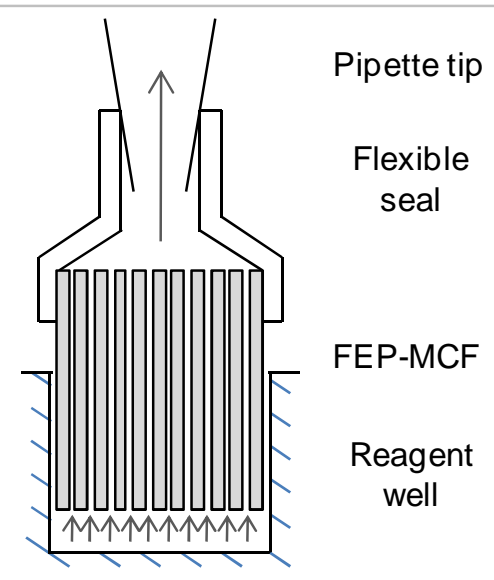

G
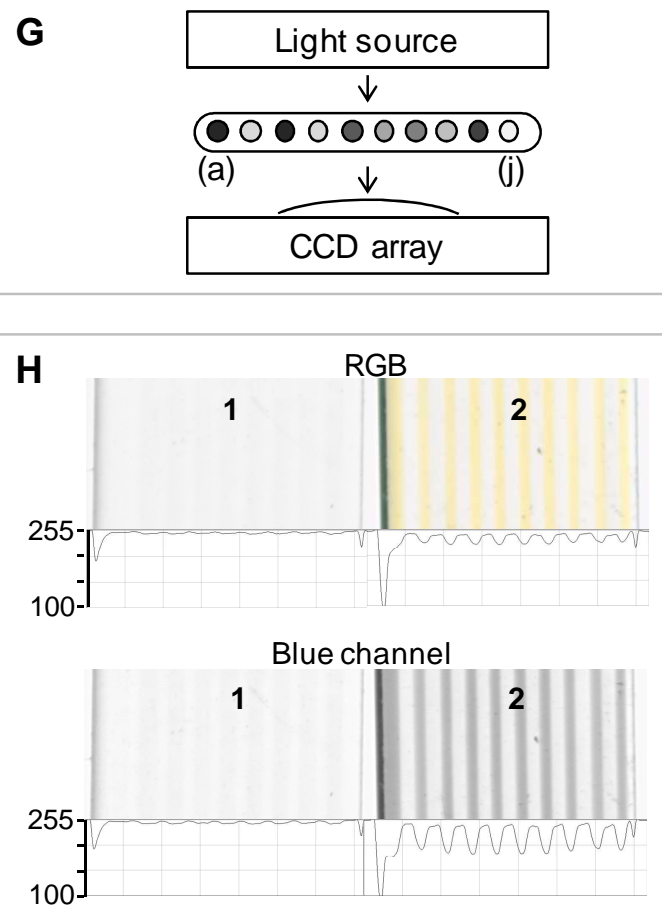

Solutions: 1 Buffer 2 Converted OPD

$\begin{array}{lllll}0 & 1 & 2 & 3 & 4 \\ & \mathrm{~mm}\end{array}$

Figure 1. Making multiplex microfluidic ELISA devices from melt-extruded fluropolymer microcapillary film

A Microcapillary film melt extruded from fluorinated-ethylene-propylene copolymer (FEP MCF). B-D Indicate the process used to make multiplex ELISA devices from FEP MCF; E-G illustrate the device fluidics and signal detection. B Coating the internal surfaces of individual capillaries within a long reel of FEP MCF with different antibodies or antigens (labelled a to j). C Cutting individual sample analysis strips from reel. D Interfacing individual sample strips with a pipettor or syringe, using silicone tubing, plus a simple reagent well to complete the microfluidic device. E Customised push-fit seal to interface MCF to syringe. F Device cross-section in use with sample strip in reagent well showing fluid flow. G Colourimetric signal detection using simple optical interrogation. H Example transmission flatbed scanner images illustrating optical clarity of FEP MCF filled either with buffer or converted substrate. RGB colour image (top) and blue channel alone (bottom) demonstrate the strong absorbance of OPD in the scanner blue channel. 
A

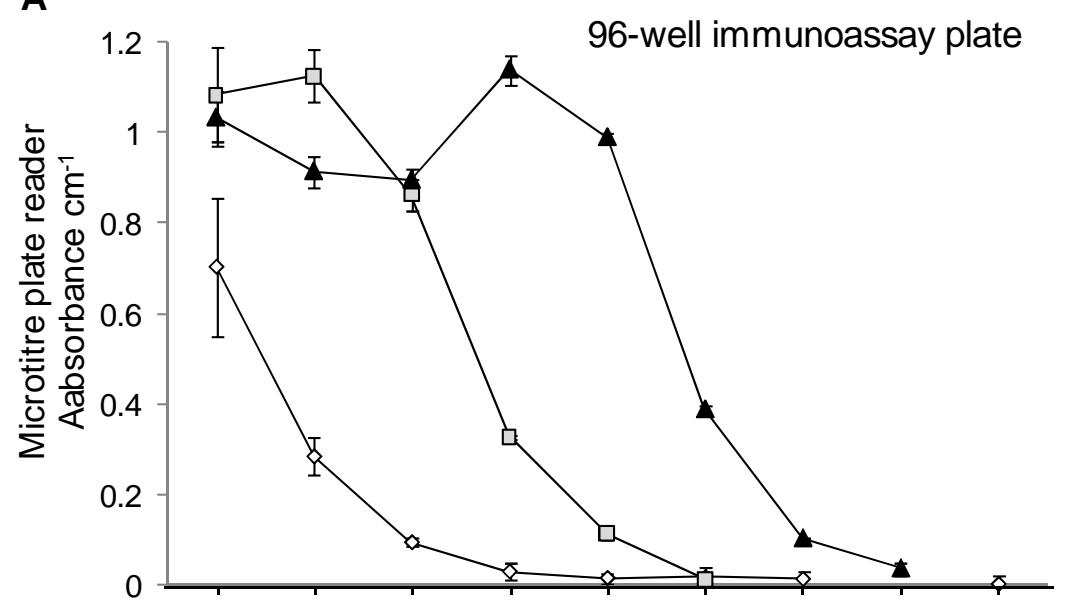

B

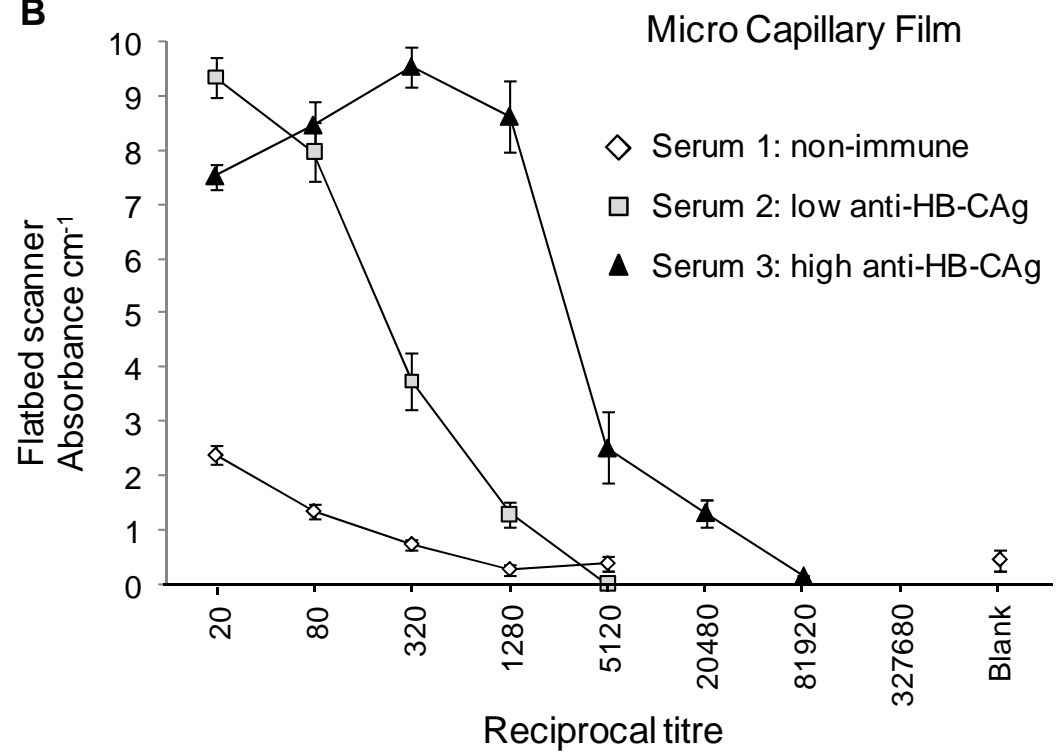

C
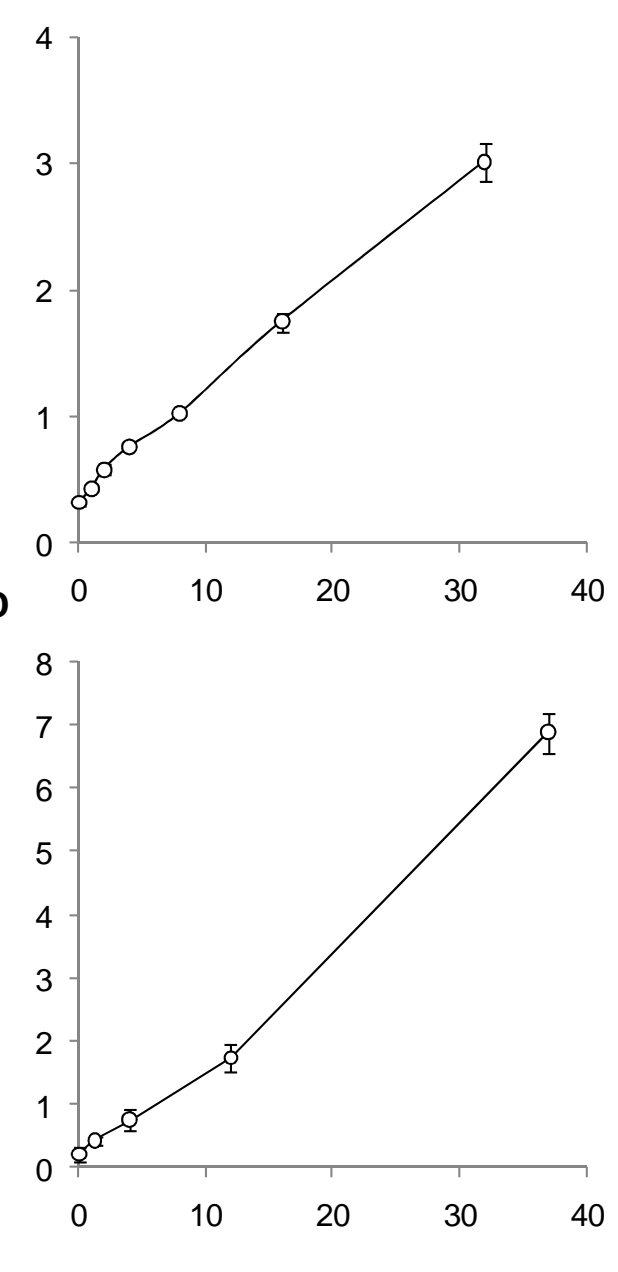

Anti-HB-CAg concentration [ng/ml]

Figure 2. Hepatitis B antibody detection comparison in FEP MCF devices vs microtitre plates.

Measurement of antibodies against HB CAg as endpoint titre in serum (A, B) and as absolute concentration (C,D) was compared using FEP MCF plus flatbed scanner (B,D) vs microtitre ELISA plate plus microtiter reader (A,C). Mouse serum samples were made with differing levels of antibodies against HB CAg by spiking control mouse serum with high or low levels of a monoclonal antibody to HB CAg. A control negative mouse serum sample or the spiked positive samples were serially diluted and tested in parallel in microtitre ELISA plates (A) or FEP MCF devices (B). A sensitivity comparison was completed by completing an ELISA on samples with the same known concentrations of a monoclonal anti-HB CAg antibody in parallel in microtitre ELISA plate (C) and FEP MCF devices (D). All error bars are shown and indicate \pm 1 standard deviation for 8 central capillaries within a single FEP MCF device, or triplicate microtitre plate wells. Data are representative of 2-3 sensitivity comparisons with similar results. 


\section{Supplementary Data}

\section{Supplementary Methods}

\section{Protein binding in capillaries within reels of FEP MCF}

Initial experiments to demonstrate the effective adsorption of antibodies or protein antigens onto the internal surface of capillaries in long reels of FEP MCF were conducted by measuring the amount of mouse IgG bound using AP-anti-mIgG and the fluorescent substrate FDP. Individual capillaries within 5 meters of FEP MCF were coated by adsorption with a series of concentrations of mouse IgG in PBS ranging from $0-100,000 \mathrm{ng} / \mathrm{ml}$, followed by washing and blocking. Replicate 50-mm long pieces taken from either end of the 5m length of FEP MCF were filled and incubated with 1:1,000 dilution of anti-mIgG-AP and incubated for 1 hour at room temperature. Each piece was then extensively washed with PBS-T and FDP buffer followed by filling with $20 \mu \mathrm{M}$ FDP substrate, incubation at room temperature for 10 minutes, and imaging with a Leica TCS SP5 broadband confocal fluorescence microscope (Leica, Milton Keynes, UK) using an excitation wavelength of $480 \mathrm{~nm}$ and measuring emission from 500 to $540 \mathrm{~nm}$. A z-stack series was scanned at different heights for each capillary and then the maximum fluorescence from each stack series projected in the z-axis using LAS AF post-processing software (Leica, Milton Keynes UK). A profile of fluorescence signal (converted to grey scale) across the MCF section was then plotted using Image J software ${ }^{1}$. The height, $h$, of the fluorescence signal within each capillary was then plotted as a function of the input concentration of mouse IgG used for coating.

\section{Comparing optical properties of MCF and individual capillaries}

MCFs containing 10 or 19 parallel microcapillaries were produced from a range of different thermoplastic materials using a novel melt-extrusion process described in patents GB2408961, EP1691964, JP2007514566 and US2009011182 and Hallmark et $a l^{2}$. Individual cylindrical FEP microcapillaries were from Upchurch Scientific (Oak Harbor, WA, USA). The optical properties of MCF fabricated from FEP and a range of other thermoplastic materials with varying optical properties (Table S1) and two sizes of commercial microcapillaries produced from FEP (Table S2) were compared. Samples were washed with PBS/ $0.05 \% \mathrm{w} / \mathrm{v}$ Tween-20 and then filled either with PBS-T or a fully-converted OPD substrate solution and scanned with a HP ScanJet 4050 Photo Scanner in transmittance mode. RGB images were acquired at a resolution of 1,200-3,200 dpi, and post-processed with Image J software ${ }^{1}$. A profile across the MCF of optical intensity was plotted for RGB images and the blue channel that showed maximum absorbance by the yellow converted OPD.

\section{Determining Absorbance in MCF from scanned images}

Scans were postprocessed using ImageJ as follows. A profile across the MCF of optical intensity averaged along a $2 \mathrm{~mm}$ section of the test strip was plotted for the blue channel that showed maximum response for the yellow coloured converted OPD substrate. Examples of the images obtained are shown in Fig. S3. A high grey level (maximum of 255) means low absorbance, whereas low grey levels (minimum of 0 ) represent high light absorbance by the solution. The signal response on each capillary was measured by taking the peak-to-valley height, $h$ from the profile plot, and then converted to absorbance by calculating Abs $=-\log ((255-h) / 255)$. The extinction coefficient measured for the flatbed scanner and microplate reader were similar (data not shown); to directly compare the signal response in the FEP MCF and microwell plates the absorbance values were normalised to units of $\mathrm{cm}^{-1}$ by dividing by the maximum path length of $0.02 \mathrm{~cm}$ for the FEP MCF capillaries or $0.3 \mathrm{~cm}$ to the microwell plate.

When all capillaries within FEP MCF strips were used to measure the same ELISA analyte (i.e. singleplex assays), the mean value for Abs/cm was taken for the 8 central capillaries; the outer two capillaries were disregarded due to their reduced diameter compared to the central 8 capillaries (caused by the extrusion process) which returned lower Absorbance values even when the same converted OPD solution was filled into all capillaries; however these channels could be utilised simply by correcting for the reduced optical pathlength. Furthermore, in some cases edge refraction by the semi-circular edges of the film caused noise that interfered with outer capillary signal detection (Fig. S2A); this could be eliminated by modifying the extrusion die geometry to separate the outer capillaries further from the sides of the film.

\section{Supplementary Results}

\section{Adsorption of proteins inside capillaries within reels of FEP MCF}

The adsorption of antibodies onto the surface of capillaries within a long reel of MCF was initially studied using a simple direct ELISA that detected bound mouse IgG using AP-anti-mIgG plus the substrate FDP (Fig. S1A). The internal capillary wall of FEP MCF shows high hydrophobicity due to the hydrophobicity of FEP combined with a high surface roughness with a contact angle with water of $123.8 \pm 1.6$ degrees estimated with Laplace-Young 
equation using hydrostatic equilibrium height. Individual capillaries were coated with varying concentrations of $\mathrm{mIgG}$ and adsorbed antibody quantified by determining peak fluorescence height (Fig. S1C). The signal was proportional to the concentration of $\mathrm{IgG}$ in the solution for concentrations below $800 \mathrm{ng} / \mathrm{ml}$, whereas for concentrations of $\mathrm{IgG}$ of $800 \mathrm{ng} / \mathrm{ml}$ or higher there was no further increase in $\mathrm{mIgG}$ on the surface of the capillary (Figs S1B,C). This pattern is characteristic of a Langmuir binding isotherm with antibody adsorption to the FEP surface in a monolayer with the plateau presumed to be due to surface saturation.

To test if antibody was depleted by adsorption along the length of a long capillary, capillaries within a 5m length of FEP MCF were coated, a piece was taken from the inlet and output, and bound $\mathrm{mIgG}$ levels was compared by quantifying fluorescent signal. Even when the input $\mathrm{mIgG}$ concentration was limiting, at $32-160 \mathrm{ng} / \mathrm{ml}$, equal fluorescence was detected at the outlet and inlet, indicating even coating along the length of the 5m capillary (Fig. S1D). These initial experiments demonstrated the ease of adsorbing antibodies and antigens inside FEP MCF, and proved the feasibility of the production method outlined in Fig. 1 for producing batches of identical devices capable of multiple analyte detection in the parallel array of capillaries. Similar adsorption was seen with other proteins, and adsorbed $\mathrm{mIgG}$ was also detected using anti-mouse HRP with the colourimetric substrate OPD imaged using a flatbed scanner (data not shown). Protein adsorption was also measured by directly coating capillary walls with enzyme-antibody conjugates, and adsorption inside the capillaries of MCF extruded from a range of different thermoplastics was observed - albeit with poor optical clarity - suggesting that protein binding did not require any particular surface characteristic of FEP (data not shown).

\section{Optical properties of FEP MCF}

The schematic cross section of FEP MCF highlights the flat top and bottom surfaces of the film (Fig. S2A top), in contrast to the individual single capillaries that have a round section (Fig. S2B, C top). From the images and intensity profiles, the FEP MCF containing buffer alone was completely transparent apart from grey lines along either edge of the film (Fig. S2A). These lines were caused by refraction of the transmitted light by the rounded edges and varied in intensity depending on positioning within the scanner; for example in Fig. S2A, the left edge appears darker than the right edge, with a wider and more intensely grey peak. When OPD was filled into all capillaries, however, intense yellow lines were seen within the film (Fig. S2A). This represented strong blue light absorbance, as seen by the dark grey lines and strong absorbance peaks in the blue colour channel (Fig. S2A, bottom). The effects of edge refraction are again clear, with the yellow signal of the outermost left capillary merging with the grey edge absorbance, although the outermost right capillary can be clearly separated from the grey edge line.

Apart from the outermost two capillaries (because of the semi-circular edges of the film), therefore, the FEP MCF has excellent transparency for optical interrogation; in contrast, when single capillaries were imaged, refraction effects from the rounded sides dominated the images (Fig. S2B, C). Firstly, for a thin walled capillary, strong grey lines were seen at both edges when filled with buffer, once again with varying intensity depending on orientation (Fig. S2B). These lines merged with the yellow OPD signal, and the peak edge refraction absorbance reached greater than $60 \%$ of the peak blue absorbance of the OPD. Note that the internal diameter of this thin-walled capillary is greater than the thick-walled capillary or the FEP MCF (Table S2), giving a greater pathlength and therefore greater blue light absorbance of OPD. In order to attempt to separate the edge refraction effect from the absorbance within the capillary, a single FEP capillary with a thicker wall was tested (Fig S2C). Although this geometry clearly separated the capillary absorbance (yellow line) from the edge refraction (grey edges), the increased thickness of FEP resulted in far higher background absorbance, thereby limiting the signal to noise ratio.

To determine the importance of the optical properties of the thermoplastic material used - as opposed to the geometry - MCF samples extruded from different transparent thermoplastics with varying optical properties (Table S1) were imaged alongside FEP MCF. Whereas FEP MCF filled with buffer was transparent (Fig S2A), other MCF with refractive indexes higher than water gave strong optical distortion, resulting in appearance of strong and variable grey lines along all the capillaries (Fig. S2D-H). Although some yellow colour is visible when these capillaries were filled with OPD, the strong intensity of grey signal resulting from refraction by the internal curved walls of the capillaries prevented accurate detection of OPD within the capillaries. This problem was not compensated by splitting RGB channels since in all cases the grey refractive signal for each capillary gave blue absorbance with buffer that was as strong as the OPD absorbance. This distortion was clearly caused by the difference in refractive index between the liquid filling the capillaries and the plastic film, since it was not observed when MCF extruded from ethylene vinyl acetate (refractive index 1.48) filled with glycerol (refractive index 1.47) was scanned. Thus EVA MCF filled with glycerol gave almost as clear images as FEP MCF filled with aqueous buffers (data not shown). 
These results clearly demonstrated that simple optical detection of colourimetric ELISA substrates by transmission imaging is feasible in FEP in the form of an MCF, but not possible either in single FEP capillaries or in MCF extruded from other thermoplastics with inferior optical properties. Three dimensional reconstruction of the capillary volume using from a z-stack also clearly demonstrated the absence of any optical distortion when imaging fluorescent dye solutions within FEP MCF using laser scanning confocal microscopy (data not shown).

\section{Cost estimates of MCF devices}

The typical density of FEP MCF is $5 \mathrm{~g} / \mathrm{m}$, with a market cost for pelleted FEP material in the range of $£ 80 / \mathrm{kg}$. Using this procedure, a 10-plex, $50 \mathrm{~mm}$ long MCF FEP test strip can be produced for less than $£ 0.10$, reduced to $£ 0.02$ if the length of test strip is further reduced to $10 \mathrm{~mm}$ (the minimum length required for signal detection is 2 $\mathrm{mm}$ as mentioned above in materials and method section). Clearly, this cost only reflects one part of complete device manufacturing; however, device cost is clearly unlikely to be limited by the manufacturing cost of this core microchannel element.

\section{Supplementary Tables}

Table S1. Geometry and properties of MCF extruded from various thermoplastic polymers.

$\begin{array}{llccc} & \begin{array}{l}\text { Thermoplastic } \\ \text { polymer }\end{array} & \begin{array}{c}\text { Mean capillary } \\ \text { diameter }(\boldsymbol{\mu m})\end{array} & \begin{array}{c}\text { Number of } \\ \text { capillaries }\end{array} & \begin{array}{c}\text { Polymer } \\ \text { Refractive } \\ \text { Index }\end{array} \\ \text { FEP MCF } & \begin{array}{l}\text { Fluorinated ethylene } \\ \text { propylene copolymer }\end{array} & 206 & 10 & 1.338 \\ \text { EVA MCF } & \text { Ethylene vinyl acetate } & 142 & 19 & 1.48 \\ \text { EVOH MCF } & \text { Ethylene vinyl alcohol } & 109 & 19 & 1.51-1.52 \\ \text { LLDPE MCF } & \begin{array}{l}\text { Linear low-density } \\ \text { polyethylene }\end{array} & 167 & 19 & 1.51 \\ \text { COW-voidage } & \begin{array}{l}\text { Cyclic olefin } \\ \text { copolymer }\end{array} & 119 & 19 & 1.53 \\ \text { LLDPE MCF } & \begin{array}{l}\text { Linear low-density } \\ \text { polyethylene }\end{array} & 200 & 13 & 1.51 \\ \text { high-voidage } & & & & \end{array}$

Table S2. Geometry of individual FEP capillaries.

$\begin{array}{llll}\text { Capillary } & \text { Material } & \text { O.D. }(\mathbf{m m}) & \text { I.D. }(\mathbf{m m}) \\ \text { FEP1-32x0.016 } & \text { FEP } & 0.794 & 0.406 \\ \text { FEP1-16x0.008 } & \text { FEP } & 1.59 & 0.203\end{array}$

\section{Supplementary references}

1. M. D. Abramoff, P. J. Magelhaes and S. J. Ram, Biophotonics International, 2004, 11, 36-42.

2. B. Hallmark, M. R. Mackley and F. Gadala-Maria, Advanced Engineering Materials, 2005, 7, 545-547. 


\section{Supplementary Figures}

A Anti-m lgG-AP
Mouse lgG coated onto surface
Surface

B

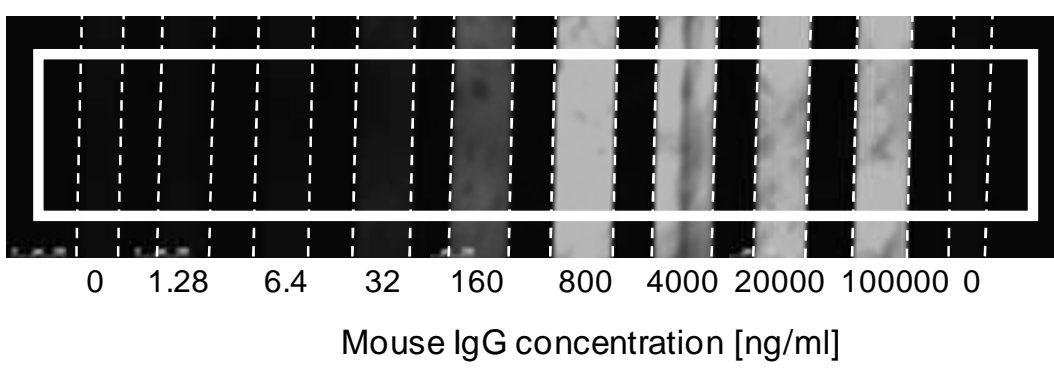

C

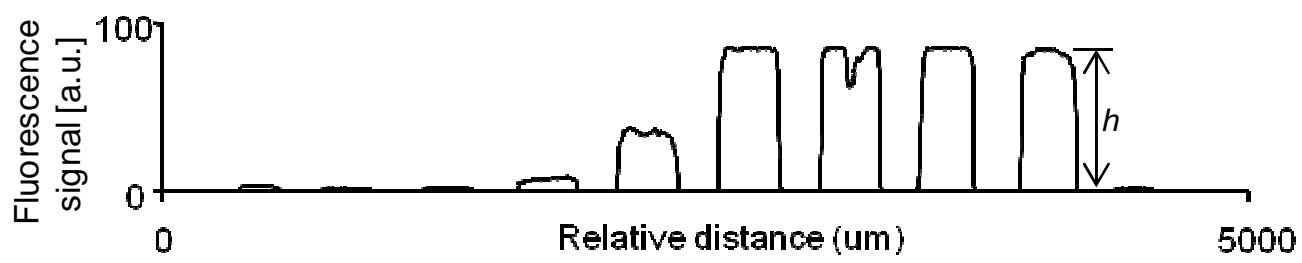

D

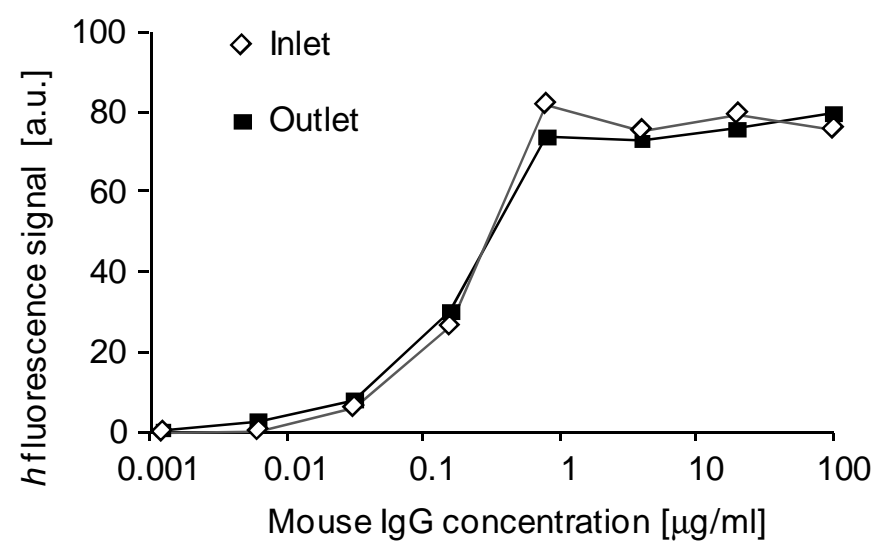

Figure S1. Evaluation of protein immobilization and signal detection within FEP MCF capillaries.

A Schematic representation of the direct assay to measure antibody immobilisation. B Fluorescence signal in a representative section of the FEP MCF, coated with increasing concentrations of mouse IgG and quantified using a confocal fluorescence microscope. C Plot of mean fluorescent intensity signal across FEP MCF. D Plot of peak height, h, of the fluorescence signal generated at each initial concentration of mouse IgG for the inlet and outlet of a $5 \mathrm{~m}$ long reel of FEP MCF indicating no depletion of the IgG along the length of the reel. This data is representative of 2 similar binding tests. 

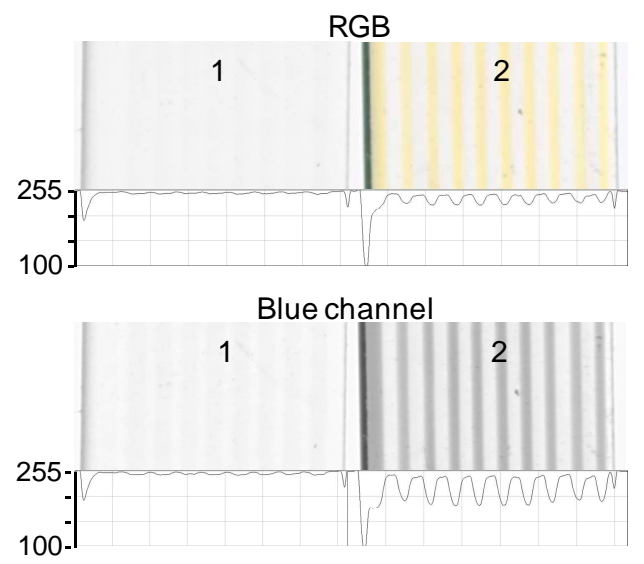

B FEP thin wall ${ }^{\text {capillary }}$
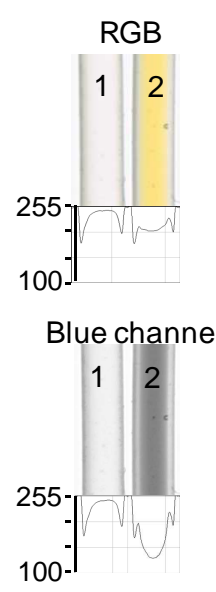
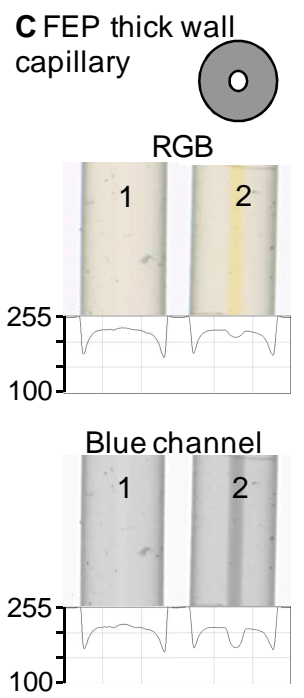

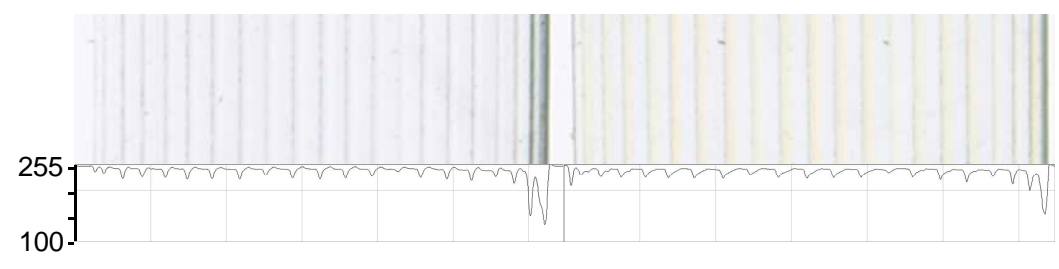

E EVOH MCF Refractive index 1.51

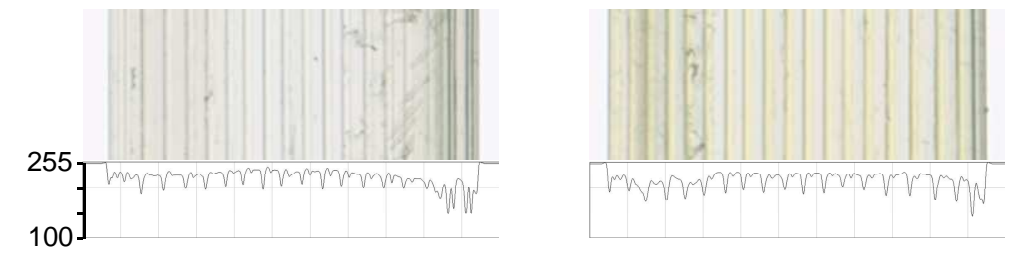

F LLDPE MCF (low-voidage) Refractive index 1.51

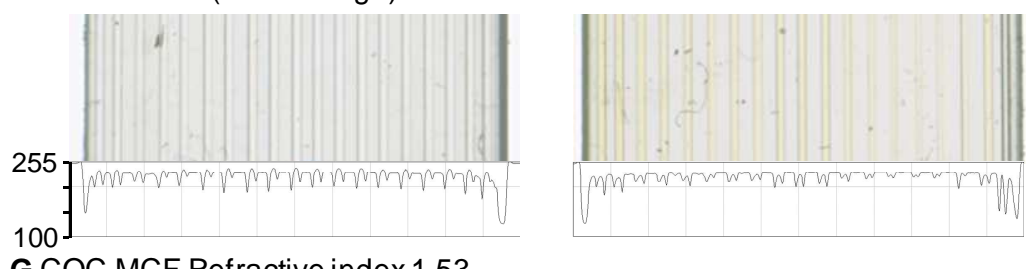

G COC MCF Refractive index 1.53
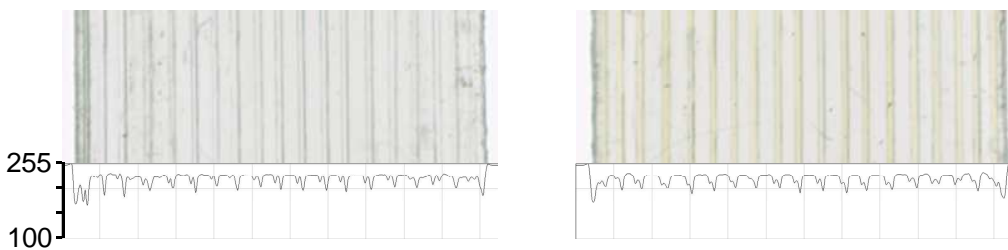

H LLDPE MCF (high-voidage) Refractive index 1.51
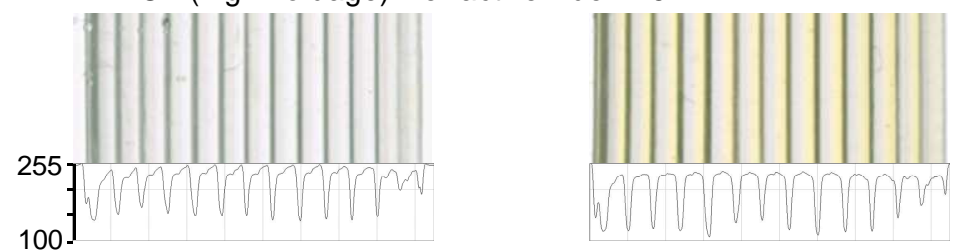

Figure S2. Comparison of optical properties of MCF extruded from FEP and other thermoplastics, and individual FEP capillaries, imaged using a flatbed scanner.

Two pieces of each material were washed in PBS-Tween solution, filled with (1) PBS-T or (2) fully converted OPD substrate and scanned in transmitted mode at 3,200 dpi with a HP ScanJet 4050 Photo Scanner. A Top: diagram representing cross section geometry of MCF. Middle: RGB image of the capillary array, showing an averaged intensity plot across the film underneath. Lower: blue channel split from RGB images, with averaged intensity ploy underneath. B and C Cross-section diagram, RGB and blue channel images and intensity plots for either thin wall, small o.d./i.d. ratio (B) or thick wall, high o.d./i.d. ratio (C) individual capillaries made from FEP. D-H Images of MCF extruded from plastics with different refractive index than water; details of MCF thermoplastic, size and optical properties are given in Table S1. 


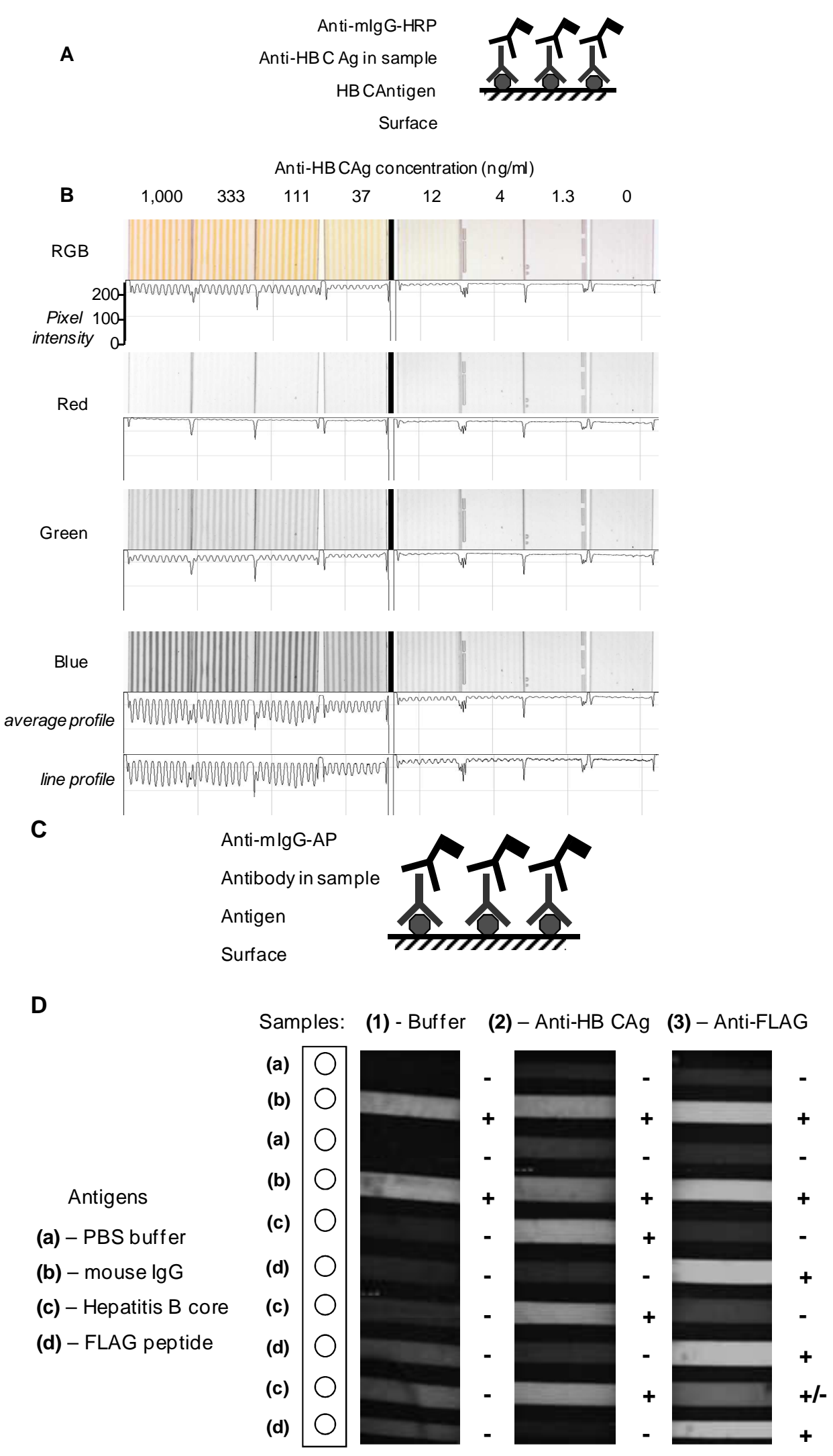

different pattern of detection channels and antigens.
Figure S3. Examples of images of singleplex and multiplex colorimetric and fluorescent ELISA conducted in FEP MCF.

(A-B) Singleplex colorimetric ELISA images in FEP MCF devices. A Assay schematic. B OPD detection in FEP MCF devices at increasing concentrations of anti-HB CAg. The top row shows the profile plot of the scanned RGB images that were then split into Red, Green and Blue channels, and the peak-height in the grey plot was used as a measure of OPD signal intensity. Data are representative of more than 4 repeat assays.

(C-D) Multiplex fluorescent ELISA in FEP MCF devices. $\mathbf{C}$ Assay schematic. D The 10 capillaries in a reel of a FEP MCF were individually coated with the following antigens: (a) negative (buffer) control, (b) positive control mouse IgG, (c) antigen FLAG peptide, and (d) Hepatitis B Core antigen, in the pattern indicated, and then trimmed in individual 50-mm sections for testing the indicated samples: (1) buffer, (2) anti-HB CAg, and (3) anti-FLAG. The fluorescence signal was imaged after 15 min by confocal microscopy. A representative plot of the fluorescence detected is shown for each sample, and each capillary is scored to indicate positive or negative signal. Similar results were seen with replicate test strips, and similar findings were observed in a repeat experiment with a 